\begin{tabular}{|l|l|l|l|}
\hline Eiszeitalter u. Gegenwart & $\mathbf{3 0}$ & $\begin{array}{c}89-100 \\
\text { 3 Abb., } 4 \text { Tab., } 1 \text { Taf. }\end{array}$ & Hannover 1980 \\
\hline
\end{tabular}

\title{
Neue Ergebnisse über das Jungquartär im Neckarschwemmfächer bei Heidelberg
}

\author{
Manfred Löscher, Bernhard Becker, Michael Bruns, Ulrike Hieronymus, \\ Roland Mäusbacher, Marianne Münnich, Klaus Münzing \& Jürgen Schedler
}

Alluvial fan, genesis, climatic effect, pollen, fossil wood, dendrochronology, C-14 dating, stratification, kryoturbation, Riss-Wuerm Interglacial, geological sestion

Upper Rhine Valley (Neckar, near Mannheim-Wollstadt), Baden-Wuerttemberg, TK 25: Nr. 6417

Kurzf a ssung: Am Beispiel eines repräsentativen Aufschlusses nordwestlich MannheimWallstadt wird aufgezeigt, wie sich mit Hilfe paläontologischer Methoden (Konchylien, 14C-Daten, Pollen- und Holzartenbestimmung) sowie Sediment- und Strukturmerkmalen (Korngröße, Schichtung, Kryoturbationserscheinungen etc.) das Jungquartär des Neckarschwemmfächers stratigraphisch, paläoklimatisch und genetisch gliedern läßt.

Das Riß-Würm-Interglazial, bisher im nördlichen Oberrheingraben nur sedimentologisch erfaßt, kann im Neckarschwemmfächer auf Grund von Eichenholzfunden und warmzeitlichen Konchylien - in wesentlich geringerer Tiefe als bisher angenommen - nachgewiesen werden.

Mittels Holzartenbestimmung läßt sich von etwa 50000 bis ca. 42500 J.v.h. ein kühl-atlantisches und von ca. 42500 bis etwa 27000 J.v.h. ein kühl-kontinentales Klima rekonstruieren. Für ein sehr kaltes Klima von 43000-39000 J.v.h., wie es in den Niederlanden von ZaGwiJn und PAEPE (1968) festgestellt wurde, ergeben sich keine Anhaltspunkte.

Das Würm-Hochglazial (oberes Pleniglazial) ist entweder nur relativ geringmächtig oder nur indirekt (durch intensive Kryoturbationserscheinungen etc.) nachweisbar.

\section{[New Results on the Upper Pleistocene Sediments in the Neckar Fan near Heidelberg]}

By means of a representative gravel pit northwest of Mannheim-Wallstadt it is shown how the late quarternary of the Neckar fan can be classified stratigraphically, paleontologically and genetically.

The Riß-Würm-interglacial, which was marked until now only by sedimentological methods, can be proved now by oak wood and interglacial molluscs in a considerably lower depth as supposed. Numerous findings of pieces of wood make it possible to prove the existence of a coniferous forest for the period between about 50000 and 27000 years BP (middle pleniglacial).

Due to the composition of its species it can be concluded that the climate was cool-atlantic from about $50000-42500$ years B.P. and cool-continental from about $42500-27000$ years B.P. There is no indication that there had been an extremely cold climate in the period between 43000 and 39000 years B.P., as it has been proved for the Netherlands by ZAGwiJn and PAEPE (1968).

The sediments of the upper pleniglacial are either thin or they can only be proved indirectly (e.g. by intensive cryoturbation phenomena).

\section{Einleitung und Problemstellung}

Das Jungquartär des Neckarschwemmfächers sowie des gesamten Oberrheingrabens ist in den letzten Jahrzehnten trotz z. T. ausgezeichneter Aufschlußverhältnisse wenig untersucht worden. Die jüngeren Publikationen über den Neckarschwemmfächer und die

*) Anschriften der Verfasser: Dr. M. Lö scher, Ulrike H i e r o n y mus und R. Mä us b a ch e r, Geogr. Institut der Universität Heidelberg, 6900 Heidelberg. - Dr. B. B e ck e r und Dipl.-Biol. J. S c h e d l e r, Botanisches Institut der Universität Hohenheim, 7000 StuttgartHohenheim. - Dipl.-Phys. M. B r u n s, Heidelberger Akademie der Wissenschaften, c/o Institut für Umweltphysik der Universität Heidelberg, 6900 Heidelberg. - Dipl.-Phys. Marianne Mü n $\mathrm{n}$ ich, ${ }^{14} \mathrm{C}$-Labor des Institutes für Umweltphysik der Universität Heidelberg, 6900 Heidelberg. - Dr. K. M ü n z ing, Geologisches Landesamt Baden-Württemberg, 7800 Freiburg. 
angrenzenden Gebiete basieren weitgehend auf der Auswertung von Tiefbohrungen ${ }^{1}$ ) und damit auf sedimentologisch-lithologischen Methoden (SCHNEIDER et al. 1975, Armbruster et al. 1977, Sidki 1976, FEZER 1974, 1977) und befassen sich vorwiegend mit der Gliederung des gesamten Quartärs, einschließlich des Jungtertiärs, und berühren das Jungquartär nur am Rande.

Bei dem Versuch, das Jungquartär stratigraphisch zu gliedern, kann man auf das stellenweise reichhaltig vorkommende und relativ gut zugängige organische Material (Hölzer, Torf, Konchylien, Säugerknochen etc.) und die in den letzten Jahrzehnten neu entwickelten bzw. verfeinerten Arbeitsmethoden ( ${ }^{14} \mathrm{C}$, Dendrochronologie etc.) zurïckgreifen.

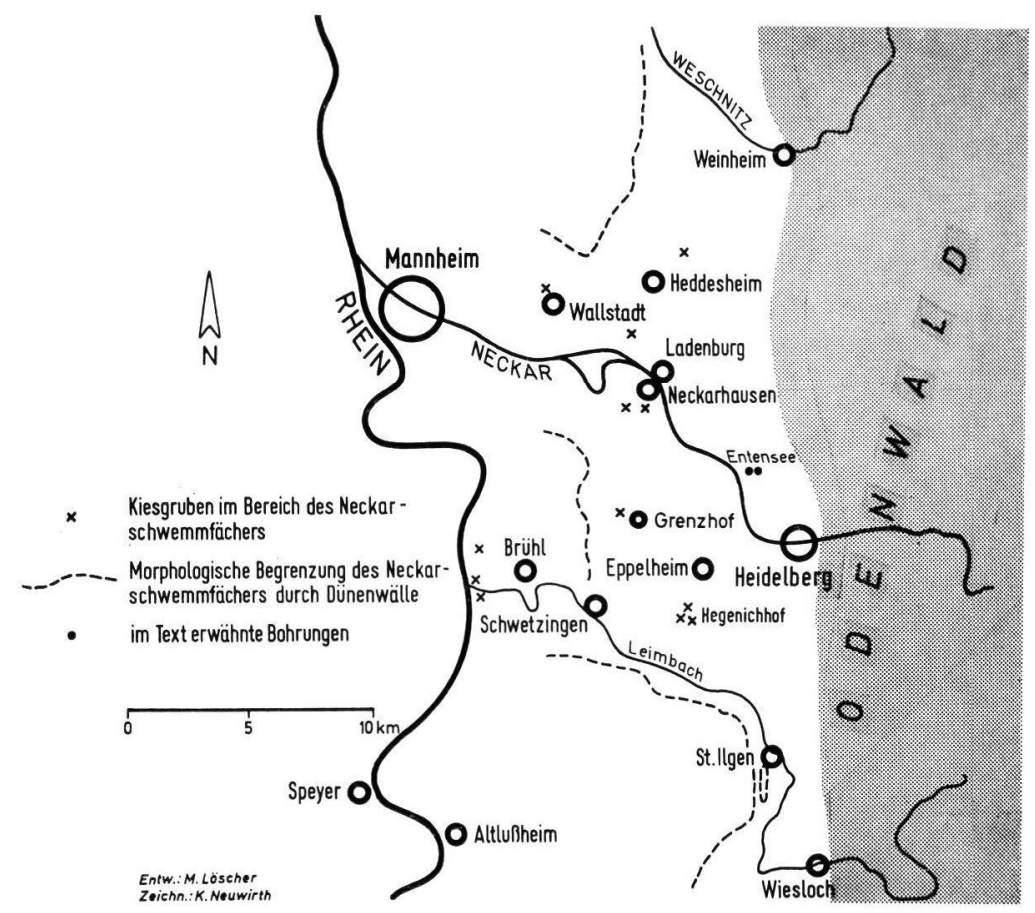

Abb. 1: Der Neckarschwemmfächer und die derzeit vorhandenen Aufschlüsse.

Nachdem 1975 in den Kiesgruben nordwestlich des Grenzhofes erstmals Hölzer entnommen wurden (Löscher 1979), erbrachte die systematische Suche bald auch in vielen anderen Aufschlüssen reichhaltiges Material.

Bei einer Vorbereitungsfahrt zur geomorphologischen Kartierung von Blatt 16417 $1: 25000$ Mannheim-Nordost ${ }^{2}$ ) der TK 25 im Rahmen des GMK-Projektes (vgl. Barsch 1976) wurden dann Anfang 1976 von Barsch, Löscher und Mäusbacher in der Kiesgrube Heckmann, NW Wallstadt, ebenfalls Holzreste geborgen und - auf schnelle Initiative von D. BARSCH - im ${ }^{14} \mathrm{C}$-Labor des Institutes für Umweltphysik der Universität

1) Diese Tiefbohrungen wurden früher für die Erdölgewinnung niedergebracht. Heute dienen sie hauptsächlich der Erfassung der Grundwasservorräte bzw. der Wasserversorgung und nur gelegentlich anderen Zwecken wie z. B. der Anlage unterirdischer Gasspeicher etc.

2) Dieses Blatt ist als Nr. 3 der Geomorphologischen Karte der Bundesrepublik Deutschland Ende 1979 erschienen. 
Heidelberg ${ }^{3}$ ) datiert. Bis Ende 1979 wurden dann in dieser Grube allein in den obersten 13,5 m (d. h. innerhalb der Horizonte IV $a+b$ und V, s. Abb. 2 und Tab. 3) 112 Hölzer geborgen und von B. Becker und U. Hieronymus nach Holzarten bestimmt. K. Münzing hat die Konchylien und J. Schedler denPollen bestimmt.

Durch die Untersuchungen der jungquartären Ablagerungen des Neckarschwemmfächers, von denen hier nur ein kleiner Teil vorgelegt wird, ergeben sich hauptsächlich zwei Problemkreise, zu denen in nächster Zeit noch mehrfach Stellung genommen werden wird:

a) jungquartäre Stratigraphie, Genese und Chronologie des Neckarschwemmfächers,

b) Chronologie und Klima der letzten Kaltzeit.

Die Klärung der genannten Probleme erfordert permanente Beobachtung über einen längeren Zeitraum und Anwendung aller zur Verfügung stehenden Arbeitsmethoden. Nur so wird es einmal möglich sein, das gesamte Spektrum des anscheinend recht differenzierten Aufbaus des Neckarschwemmfächers und seiner benachbarten Gebiete einigermaßen zu erfassen $^{4}$ ).

\section{Beobachtungen und Ergebnisse aus der Kiesgrube Heckmann, nordwestlich Mann- heim-Wallstadt (TK 25, Blatt 6417 Mannheim-Nordost; R 3466900 / H 5485300)}

In diesem Aufschluß wird das Sediment bis max. $33 \mathrm{~m}$ unter Flur abgebaut; davon liegen ca. $23 \mathrm{~m}$ unter dem Grundwasserspiegel. Einen Überblick der oberhalb des Grundwasserspiegels liegenden Schichten gibt Abb. 2. Im folgenden werden die einzelnen Schichtpakete beschrieben sowie genetisch und stratigraphisch gedeutet.

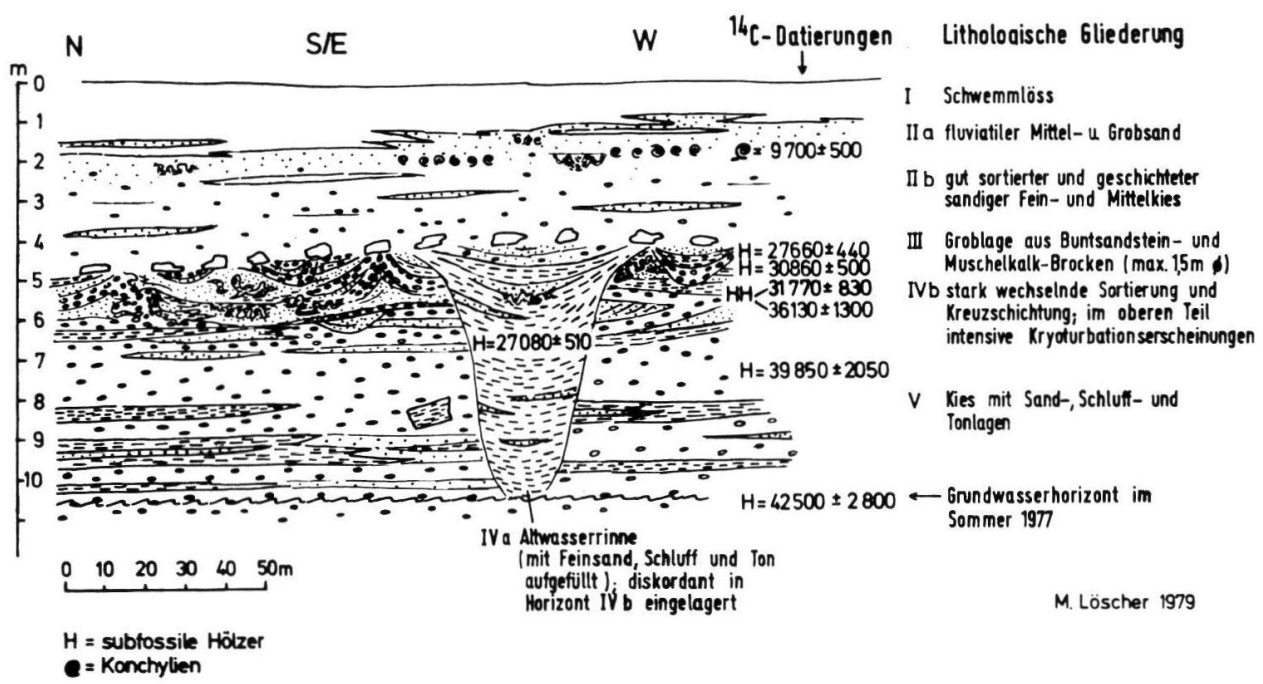

Abb. 2: Profil der Kiesgrube Heckmann nordwestlich Mannheim-Wallstadt (R 3466900/H 5485300).

Schicht I besteht aus 1-1,5 m Schwemmlöß, der im Bereich des Aufschlusses stellenweise als schwarzerdeartiger Boden ausgebildet ist und in dem nach unten hin in zunehmendem Maße schluffige Feinsandstreifen eingelagert sind. Der Schwemmlöß wird -

3) Im Rahmen des Forschungsvorhabens „radiometrische Altersbestimmung von Wasser und Sedimenten der Heidelberger Akademie der Wissenschaften".

4) Für fachliche Beratung, Diskussionsbeiträge und Durchsicht des Manuskripts danken wir Prof. Dr. D. Barsch, Prof. Dr. F. Fezer, Dr. A. Zienert. 
z. T. schon seit der geologischen Kartierung des Gebietes (um 1900 und früher) - als frühholozänes Hochflutsediment des Neckars angesehen (SchотTLER 1906).

Schicht II beginnt im Hangenden zunächst mit ca. 0,5 $\mathrm{m}$ fluvialem Fein- und Mittelsand, der sich nach oben hin mit dem Schwemmlöß verzahnt und nach unten in $3-4 \mathrm{~m}$ sehr gut geschichteten und sortierten, sandigen Fein- und Mittelkies übergeht. Im oberen Teil wurden vereinzelt Kryoturbationserscheinungen gesichtet. Vor allem im hangenden Sand und in den oberen Kieslagen dieser Schicht findet man Linsen mit zahlreichen Konchylien (artenarm, individuenreich), die durch fast $2 \mathrm{~cm}$ große Exemplare von Helicigona arbustorum und Succinea putris gekennzeichnet sind. Eine ${ }^{14} \mathrm{C}$-Analyse einer sehr sorgfältig gereinigten Probe solcher Konchylienschalen ergab ein Alter von $9700 \pm 500$ Jahren $^{5}$ ) (s. Abb. 2).

Tab. 1: Konchylien (nur Landschnecken) aus der Kiesgrube Heckmann, nordwestlich MannheimWallstadt, und ihre klimatische Deutung. (Die Bestimmung erfolgte durch K. Münzing. Die vollständigen Faunenlisten werden zu einem späteren Zeitpunkt veröffentlicht.) Besonders bemerkenswert ist das Auftreten von Vallonia tenuilabris und Helicopsis striata (wenige Exemplare in Proben 1-4). Beide Arten waren bisher aus sicher würmzeitlichen Ablagerungen der badischen und elsäßischen Rheinebene unbekannt.

\begin{tabular}{|c|c|c|c|c|}
\hline $\begin{array}{l}\text { Probe } \\
\text { Nr. }\end{array}$ & $\begin{array}{l}\text { Entnahme- } \\
\text { tiefe } \\
\text { (in } \mathrm{m} \\
\text { unter Flur) }\end{array}$ & $\begin{array}{l}\text { Zahl der } \\
\text { bestimmten } \\
\text { Exemplare } \\
\text { (Arten) }\end{array}$ & $\begin{array}{l}\text { klimatische } \\
\text { Deutung } \\
\text { (nach MüNZING) }\end{array}$ & $\begin{array}{l}\text { charakteristische Vertreter } \\
\text { (Anzahl in Klammern) }\end{array}$ \\
\hline 1 & 1,5 & $247(14)$ & kaltzeitlich & Vallonienfauna \\
\hline 2 & 2 & $813(16)$ & $\begin{array}{l}\text { spätkaltzeitlich } \\
\text { - frühholozän }\end{array}$ & $\begin{array}{l}\text { Vallonienfauna mit } \\
\text { Vallonia costata }(352) \\
\text { Vallonia pulchella }(49)\end{array}$ \\
\hline 3 & 3,4 & $232(11)$ & kaltzeitlich & \\
\hline 4 & 3,8 & $315(14)$ & kaltzeitlich & \\
\hline 5 & 5,3 & $760(18)$ & hochkaltzeitlich & $\begin{array}{l}\text { Columella columella (2) } \\
\text { Vallonia tenuilabris (2) }\end{array}$ \\
\hline 6 & 6,6 & $667(15)$ & hochkaltzeitlich & $\begin{array}{l}\text { Columella columella (3) } \\
\text { Pupilla loessica (3) } \\
\text { Vallonia tenuilabris }\end{array}$ \\
\hline 7 & 9 & $1579(7)$ & hochkaltzeitlich & Columella columella (93) \\
\hline 8 & $28-33$ & $331(19)$ & warmzeitlich & $\begin{array}{l}\text { Cocblodina laminata (4) } \\
\text { Ena montana (1) } \\
\text { Aegopinella sp. } \\
\text { (nitenslminor) (17) }\end{array}$ \\
\hline 9 & $28-33$ & $90(11)$ & warmzeitlich & $\begin{array}{l}\text { Cocblodina laminata (1) } \\
\text { Aegopinella sp. } \\
\text { (nitens/minor) (2) }\end{array}$ \\
\hline
\end{tabular}

Aus Schicht II wurden insgesamt drei Konchylienproben entnommen und von K. Münzing als kaltzeitlich bestimmt (Proben Nr. 1-3 in Tab. 1).

Sedimentstrukturen und Fauna belegen also ziemlich sicher, daß die Schicht II auch in ihren oberen Teilen noch unter kaltzeitlichen Verhältnissen abgelagert worden ist.

Schicht III besteht im wesentlichen aus einer Lage von meist nur sehr schwach kantengerundeten Buntsandsteinblöcken (max. $1 \mathrm{~m} \phi$ und plattigen Muschelkalkblöcken (max. $1 \mathrm{~m} \varnothing$ ). Stellenweise fächert sich diese Schicht in mehrere Einzellagen von insgesamt $1 \mathrm{~m}$ Mächtigkeit auf. Dieser Blockhorizont lagert mit deutlicher Diskordanz auf den mit IV $\mathrm{a}+\mathrm{b}$ bezeichneten Schichten. Es stellt sich die Frage, ob es sich um

5) Korr. auf (85 \pm 5$) \%$ Anfangsgehalt; 14 C-Alter bei 100\% Anfangsgehalt $=11000$ \pm 100 Jahre. 
a) ein Steinpflaster handelt, das nach Auswaschung einer ursprünglich mehrere Meter mächtigen Kiesschicht übrigblieb oder

b) um ein außergewöhnliches fluviales Ereignis mit hoher Transportleistung oder

c) um eine besondere Epoche, in der die Blöcke - weitgehend eingefroren in Eisschollen - herantransportiert wurden.

Da die Ecken bei der überwiegenden Mehrzahl der Blöcke nur wenig abgerundet sind, ist die unter c) genannte Möglichkeit die wahrscheinlichste. Bei a), aber auch bei b), müßten alle Blöcke eine stärkere Zurundung zeigen. Da derartige Blockhorizonte in ähnlicher Lage auch in anderen Kiesgruben festgestellt werden, erhebt sich damit weiter die Frage nach den Möglichkeiten eines lithographisch-stratigraphischen Vergleichs solcher Horizonte.

Schicht IV b ist ca. $2 \mathrm{~m}$ mächtig und weist bei starkem Wechsel in der Korngrößensortierung (Ton, viel Schluff und Sand, Kies und vereinzelt Blöcke) auch eine starke Kreuzschichtung auf. Auffallend sind die zahlreichen kleinen Rinnenbildungen von oft nur wenigen Metern im Querschnitt. Die obere Hälfte der Schicht IV b zeigt stellenweise intensive Kryoturbationserscheinungen, die an der Grenze zu Schicht III mit scharfer Diskordanz enden (Taf. I, Fig. 1). In dieser Schicht - etwa von der Untergrenze der Kryoturbationserscheinungen an nach unten - sind Hölzer eingelagert, die max. $35 \mathrm{~cm} \phi$ (ohne Rinde und erkennbares Splintholz) erreichen (Taf. I, Fig. 2). Sie sind alle mehr oder weniger stark zersetzt, und eine Holzartenbestimmung ist schwierig. Nach der Untersuchung von 35 Proben durch B. Becker und U. Hieronymus ist jedoch gesichert, daß es sich fast ausschließlich um Nadelhölzer handelt (s. Tab. 3).

Betrachtet man nun die ${ }^{14} \mathrm{C}$-datierten Hölzer in Abbildung 2, so zeigt sich eine deutliche Übereinstimmung von chronostratigraphischer und lithostratigraphischer Abfolge.

Tab. 2: 14C-Daten aus der Kiesgrube Heckmann, nordwestlich Mannheim-Wallstadt. (Die Probe Hv 8687 wurde im 14C-Labor des Niedersächsischen Landesamtes für Bodenforschung, alle anderen im ${ }^{14} \mathrm{C}$-Labor des Institutes für Umweltphysik der Universität Heidelberg bestimmt.)

\begin{tabular}{|c|c|c|c|c|}
\hline \multicolumn{2}{|c|}{ Proben-Nr. } & Entnahmetiefe & 14C-Alter & Material \\
\hline 1) & H $5327-4893$ & 2 & $9700 \pm 500$ & Konchylien \\
\hline 2) & Hv 8687 & 6,5 & $27080 \pm 510$ & Pflanzenhäcksel \\
\hline 3) & H $5516-5051$ & 4,4 & $27660 \pm 440$ & unbekannt ${ }^{6}$ ) \\
\hline 4) & $\mathrm{H} \quad 4753-4139$ & 4,8 & $30860 \pm 500$ & Pinus sylvestris \\
\hline & $\mathrm{H} \quad 4638-4064$ & 5,3 & $31770 \pm 830$ & Abies \\
\hline & $\mathrm{H} \quad 4637-4067$ & 5,5 & $36130 \pm 1300$ & Laubholz, unbestimmbar \\
\hline & H $4754-4135$ & 7,3 & $39850 \pm 2050$ & Pinus sylvestris \\
\hline & H 4756-4136 & 10,6 & $42500 \pm 2800$ & Pinus sylvestris \\
\hline
\end{tabular}

Überraschenderweise gedieh also im Oberrheingraben während des Mittelwürms über Zehntausende von Jahren ein Wald, in dem die Kiefer eindeutig dominierte (s. Tab. 3).

Die obersten Hölzer in Schicht IV b zeigen einen kleineren Durchmesser (max. 4-5 $\mathrm{cm}$ ) als weiter unten. Dies und die kräftigen Kryoturbationserscheinungen unmittelbar darüber, sowie das Vorkommen hochkaltzeitlicher Konchylien (s. Probe 5 in Tab. 1) deuten auf zunehmend kälteres Klima hin. Ob es sich jedoch schon um hochkaltzeitliches Klima handelt, ist aus mehreren Gründen fraglich. Die Diskrepanz zwischen den (kühl-temperier-

6) Die gesamte Holzsubstanz wurde zur ${ }^{14} \mathrm{C}$-Datierung benötigt. 


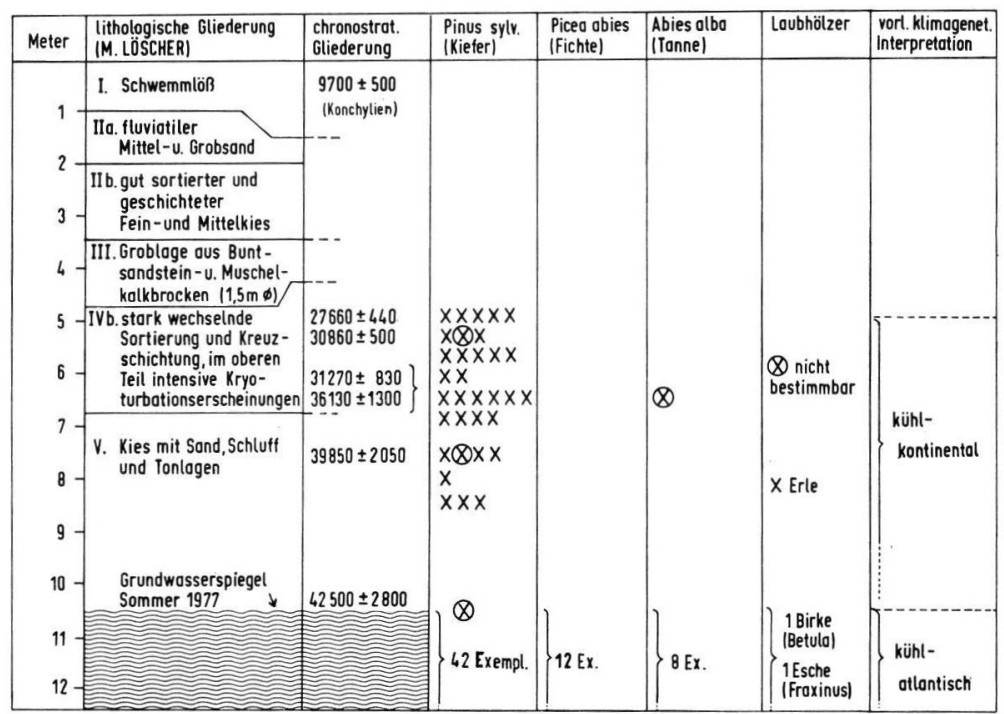

Tab. 3: Die subfossilen Hölzer aus der Kiesgrube Heckmann (nordwestlich Mannheim-Wallstadt) von $5-13 \mathrm{~m}$ unter Flur. (Bestimmung durch U. Hieronymus, unter Mithilfe von B. BeCKer). Es wurden insgesamt 112 Hölzer untersucht, von denen 3 nicht zu bestimmen waren. 7 Hölzer (5 Pinus sylvestris, 1 Picea, 1 Betula) wurden auf der Halde (ca. 6-10 m unter Flur) gefunden und nicht in die Tabelle übernommen. Die mit $(\times)$ gekennzeichneten Proben wurden ${ }^{14} \mathrm{C}$-datiert.

ten) Hölzern und den hochkaltzeitlichen Konchylien in Schicht IV b und V (Proben 5-7 in Tab. 1) kann verschiedene Ursachen haben:

a) die Fauna lebte nur teilweise am Ablagerungsort. An den Hängen des Odenwaldes bzw. in Teilen des Neckar-Hinterlandes herrschte vielleicht bereits Tundra, während in der milderen Rheinebene noch ein Baumbestand aushalten konnte.

b) die Schalen entstammen einem fossilen Spülsaum.

Die Kryoturbationserscheinungen selbst können schließlich durchaus als eine hochkaltzeitliche Überprägung angesehen werden, die den oberen Teil der Schicht IV b zu einem späteren Zeitpunkt, also im Würm-Hochglazial, erfaßt hat.

Schicht IVa ist eine mit Ton, Schluff und vereinzelten Feinsandlagen verfüllte Altwasserrinne, die diskordant in die Schichten IV b und V eingeschnitten ist und bis unter den Grundwasserspiegel reicht. Sie enthält zahlreiche Lagen Pflanzenhäcksel und vereinzelt dünne Holzreste $(\max .5 \mathrm{~cm} \phi)$. Eine ${ }^{14} \mathrm{C}$-Datierung ergab $27080 \pm 500$ Jahre und bestätigte damit den lithologisch-strukturellen Befund (s. Abb. 2).

Der Pollen aus dieser Rinnenfüllung wurde von J. SchedLer untersucht. Die Pollendichte erwies sich trotz mehrmalig aufeinanderfolgender Schweretrennung als so gering, daß die Anfertigung eines Pollendiagramms nicht in Frage kam. Die große Homogenität des Pollenspektrums gestattet es jedoch, die Werte der 27 Einzelproben zu summieren, prozentual zu berechnen und tabellarisch zu ordnen (Tab. 4).

Die Pollenerhaltung war gut, jedoch ist auf Grund der verhältnismäßig hohen Dichte von Polypodiaceen-Sporen eine Zersetzung und Umlagerung nicht auszuschließen.

Im Baumpollen-Anteil überwiegen Pinus (62,3\%) und Betula (21,4\%), gefolgt von Picea $(6,1 \%)$ und Corylus $(5,1 \%)$. Die wenigen weiteren Baumarten sind nur in Spuren vertreten und irrelevant. Wegen des beachtlichen Anteils an Nichtbaumpollen (35,5\% 
Tab. 4: Pollenspektrum aus der fossilen Altwasserrinne (Schicht IV a) in der Kiesgrube Heckmann (nordwestlich Mannheim-Wallstadt). Analyse: J. SchedLer, 1978.

\begin{tabular}{|c|c|c|c|c|}
\hline \multirow{16}{*}{$\begin{array}{l}\text { Proben } \\
1-27 \\
\text { à } 10 \mathrm{~cm}\end{array}$} & \multicolumn{2}{|c|}{$\begin{array}{l}\text { B a um polle } \mathrm{m} \\
64,5 \%\end{array}$} & \multicolumn{2}{|l|}{$\begin{array}{l}\text { Nich t b a m pollen } \\
35,5 \%\end{array}$} \\
\hline & Pinus & 62,3 & Caryophyllaceae & 9,2 \\
\hline & Picea & 6,1 & Artemisia & 7,1 \\
\hline & Abies & 3,1 & Polygonum bistorta-Typ & 6,1 \\
\hline & Betula & 21,4 & Rubiaceae & 5,1 \\
\hline & Corylus & 5,1 & Plantago lanceolata-Typ & 4,1 \\
\hline & Alnus & 1,0 & Thalictrum & 4,1 \\
\hline & Fagus & 1,0 & Ranunculaceae & 2,0 \\
\hline & & & Epilobium & 2,0 \\
\hline & & & Asteraceae & $\begin{array}{l}2,0 \\
2,0\end{array}$ \\
\hline & Sporen & & $\begin{array}{l}\text { Denopodiaceae } \\
\text { Dipsacaceae }\end{array}$ & 2,0 \\
\hline & & & Rosaceae s.1. & 1,0 \\
\hline & Polypodiaceae & 19,1 & Sanguisorba offic.-Typ & 1,0 \\
\hline & Polypodium & 1,0 & Cichoriaceae & 1,0 \\
\hline & Cystopteris & 0,5 & Gramineae & 5,1 \\
\hline & Sphagnum & 1,0 & Cyperaceae & 1,0 \\
\hline
\end{tabular}

Angaben in \% berechnet auf Baumpollen-Summe

excl. Sporen) mit 16 aufgefundenen Taxa, insbesondere Caryophyllaceen, Artemisia, Polygonum bistorta-Typ, Thalictrum und Gramineen, muß man auf einen offenen Vegetationstyp schließen.

Offensichtlich handelt es sich bei der Rinnenfüllung um ein Sediment, das unter zunehmend kälteren Klimabedingungen abgelagert wurde, etwa kurz vor dem Eintreten des Würm-Hochglazials.

Schicht V gleicht in Korngröße und Sortierung dem unteren Teil von Schicht II, jedoch sind zusätzlich zahlreiche Feinsand-, Schluff- und Tonbänke eingelagert; vereinzelt treten im Kies auch eckige Schluffschollen bis $2 \mathrm{~m}$ Länge auf, die wohl in dieser Form nur in gefrorenem Zustand transportfähig waren.

Auf Grund der Holzfunde ergibt sich eine ähnliche klimatische Aussage wie für den unteren Teil von Schicht IV b, also kühl-kontinental. Da der unterste Teil der über dem Grundwasser liegenden Schichten meist durch eine Halde von nachrieselndem Kies und Sand verhüllt war, konnten hieraus keine Hölzer geborgen werden.

Die Schichten unter dem Grundwasserspiegel werden bis zu einer Tiefe von max. $23 \mathrm{~m}$ ausgebaggert. Dabei kommt ebenfalls viel organisches Material — vor allem Hölzer zum Vorschein. Auf Grund ständiger Beobachtungen und Gespräche mit erfahrenen Baggerführern sind folgende Aussagen gesichert:

a) Die Schicht von 10,5-13,5 m unter Flur, also 0-3 m unter dem Grundwasserspiegel, wird häufig mit einem an Land stehenden Bagger herausgeholt und kann deshalb gut von den tieferen Schichten unterschieden werden. Die dort gefundenen Hölzer $(>42500 \pm 2800$ J.v.h.) zeigen eine etwas andere Florenzusammensetzung als bei den Schichten IV b und V (s. Tab. 3). Zwar dominiert auch hier die Kiefer, jedoch bezeugen Tanne und 1 Esche ein milderes Klima, das man am besten als kühl-atlantisch bezeichnen könnte. 
b) Die Schichten von 13,5-28 m unter Flur werden ausschließlich von einem Schwimmbagger gefördert. Das laufende Nachrutschen von Sediment im Wasser macht eine Aussage über die lithologische Abfolge in diesem Bereich fast unmöglich. Bei $57 \mathrm{Holz}-$ proben, die aus diesen Schichten geborgen und von B. BECKER untersucht wurden, ergab sich wieder eine etwas andere Artenzusammensetzung. Man kann sie - ebenso wie die in Schicht IV b und V - einem insgesamt kühl-kontinentalen Klima zuordnen.

$\begin{array}{lrll}\text { Picea } & 31 \text { Stück }(54 \%) & \text { Juniperus } & 4 \text { Stück }(7 \%) \\ \text { Pinus } & 12 \text { Stück }(21 \%) & \text { Salix } & 1 \text { Stück }(2 \%) \\ \text { Betula } & 9 \text { Stück }(16 \%) & & \end{array}$

Bis jetzt wurden in diesem Schichtpaket - trotz ständiger Beobachtung - keine wärmeliebenden Hölzer gefunden.

c) In der Schicht von ca. 28-33 m unter Flur sind bis jetzt, neben zahlreichen Nadelhölzern, mehrfach je 1-2 Eichenholz-Bruchstücke gefunden worden. Zusammen mit dem Vorkommen warmzeitlicher Konchylien (Proben 8 u. 9 in Tab. 1) ergibt sich damit ein deutlicher Beweis für warmzeitliche Flora und Fauna. Unter Berücksichtigung der noch überzeugenderen Fakten, die in einigen anderen Kiesgruben des Neckarschwemmfächers (sowie benachbarter Gebiete) gefunden wurden, kann es sich hierbei nur um Ablagerungen aus dem Riß-Würm-Interglazial handeln ${ }^{7}$ ).

Damit wäre nicht nur im Neckarschwemmfächer, sondern u. W. in der gesamten nördlichen Oberrheinebene das Riß-Würm-Interglazial zum ersten Mal mit paläontologischen Methoden nachgewiesen worden.

\section{Die Bedeutung der Daten für die Genese und Chronologie des Neckarschwemmfächers}

Die Auswertung des in der Kiesgrube Heckmann gefundenen Materials macht folgende Aussagen möglich:

a) Stammreste bis $35 \mathrm{~cm} \phi$ von Nadelhölzern und kälteresistenten Laubhölzern in den Schichten 5-13 m unter Flur belegen für die Zeit von etwa 500008)-27000 J.v.h. für den Bereich des Neckarschwemmfächers einen Nadelwald und ein kühl-kontinentales bis kühl-atlantisches Klima.

b) Das Hochwürm (also der Zeitabschnitt von etwa 25000-15000 J.v.h.) ist - wenn man die Kryoturbationserscheinungen in Schicht IV b nur als hochkaltzeitliche Uberprägung ansieht - in diesem Aufschluß kaum konkret faßbar. Vermutlich sind die hochwürmzeitlichen Sedimente am Ende des Hochwürm oder zu Beginn des Spätwürm durch Erosion bis auf ein zurückbleibendes Blockpflaster (Schicht III), das eventuell zusätzlich noch durch zahlreiche kantige Driftblöcke angereichert wurde, vollkommen ausgeräumt worden. Es ergeben sich auf jeden Fall Parallelen zu anderen Aufschlüssen des Neckarschwemmfächers, z. B. zu den Kiesgruben nordwestlich des Grenzhofes (vgl. Löscher 1979).

c) Bei ca. $28 \mathrm{~m}$ unter Flur beginnen warmzeitliche Sedimente, die nach jetzigem Stand zum Riß-Würm-Interglazial gehören. Sie reichen anscheinend mindestens bis zur max. Abbaustufe (derzeit bei $33 \mathrm{~m}$ unter Flur).

d) Auf Grund der datierten Hölzer ergibt sich für die direkt über dem Grundwasserspiegel anstehenden Schichten (Schicht V + IV b) eine Sedimentationsgeschwindigkeit von $0,4 \mathrm{~m}$ pro Jahrtausend. Diese Rate liegt zwischen der von FEZER (1977) im Gewann

7) Bisher wurde der in zahlreichen Bohrungen angetroffene, etwa $50-60 \mathrm{~m}$ unter Flur gelegene „obere Ton“ als riß-würminterglaziales Sediment angesehen (SCHNEIDER et al., 1975; ARMBRUSTER et al., 1977).

8) Durch Interpolation ermittelter Wert (s. auch Abb. 2). 
Entensee (nordwestlich Heidelberg) - auf Grund lithologischer Auswertungen - mit $1 \mathrm{~m} / 10^{3}$ a rekonstruierten und der von Löscher (1979) in den Kiesgruben nordwestlich des Grenzhofes mittels (radiocarbon datierten) subfossilen Hölzern errechneten Sedimentationsgeschwindigkeit von $0,25 \mathrm{~m} / 10^{3} \mathrm{a}$.

\section{Einordnung der Ergebnisse in die Chronologie der letzten Eiszeit}

ZaGwijn \& PAepe (1968) haben den Ablauf der letzten Eiszeit für den Bereich der Niederlande in einer inzwischen häufig zu Vergleichen herangezogenen Klimakurve dargestellt (s. Abb. 3). Dieser Klimagang ist durch zahlreiche Untersuchungen in vielen Teilen Europas immer wieder - zumindest in den Grundzügen - bestätigt worden und kann deshalb wohl auch für die Untersuchungen im Neckarschwemmfächer als geeigneter Vergleich herangezogen werden (vgl. z. B. auch FLIRI et al. 1970; HANNSs 1973; SCHLÜCHTER 1973; Grootes 1977; Hauber \& Barsch 1977; Garnes 1978 und andere).

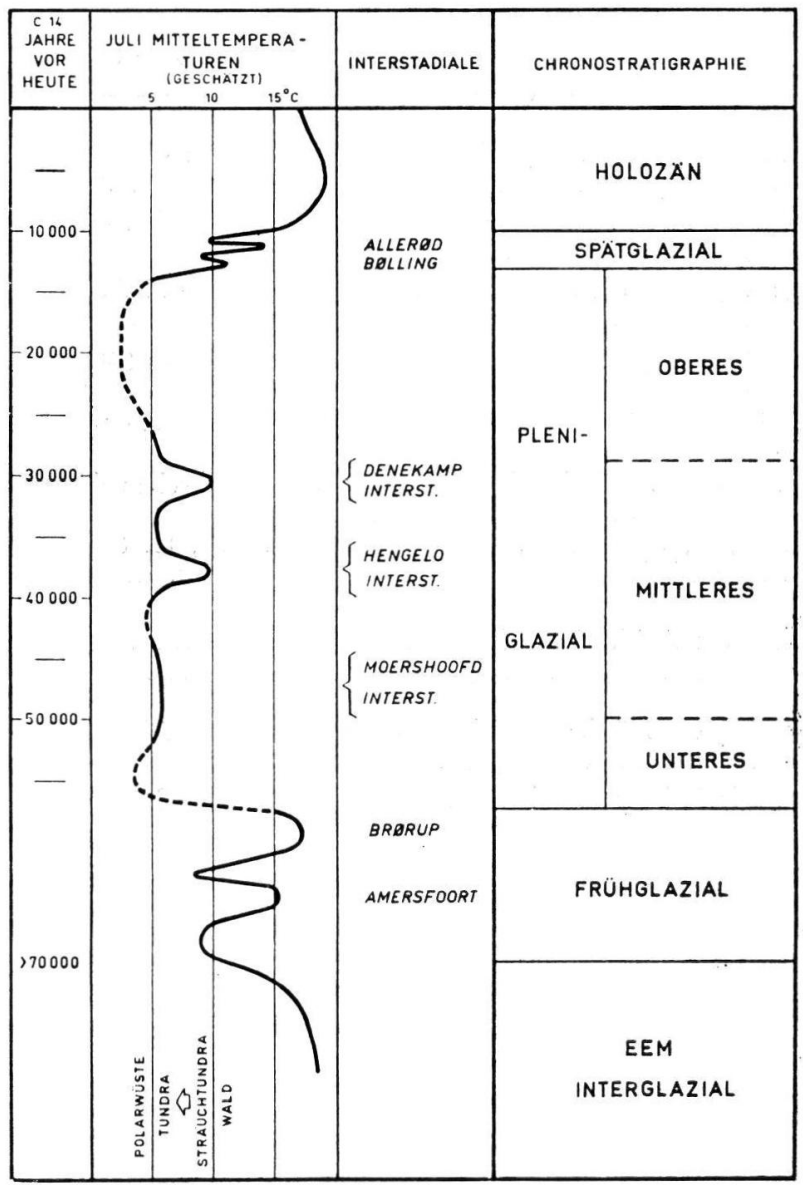

Abb. 3: aus ZAGwijn \& PAEPE (1968, Abb. 3): Klimakurve und Chronostratigraphie in der letzten Eiszeit in den Niederlanden. 
Die bisher datierten Hölzer aus dem Neckarschwemmfächer sind demnach in das mittlere Pleniglazial (etwa identisch mit dem Mittel-Würm bei Gross 1964, Abb. 1) zu stellen. Sie bestätigen die für die Niederlande konstruierte Klimakurve in den Grundzügen. Für kleinere Zeitabschnitte ergibt sich allerdings keine Deckung. So nehmen ZaGwIjN \& PAEPE von 43000-39000 J.v.h. für die Niederlande Polarwüste mit Julitemperaturen von $5^{\circ} \mathrm{C}$ an. Diese Annahme wurde bisher indirekt dadurch bestätigt, daß aus dem mittleren und nördlichen Europa für diesen Abschnitt recht wenige Datierungen vorlagen. Die Profile nordwestlich des Grenzhofes (vgl. Löscher 1979) und von Mannheim-Wallstadt belegen nun für den Neckarschwemmfächer (und damit wahrscheinlich für den gesamten Oberrheingraben) in diesem Zeitabschnitt einen Nadelwald und damit ein etwas wärmeres Klima, als es in den genannten Klimakurven zum Ausdruck kommt.

Durch gezielte Probeentnahmen und möglichst vielseitige Untersuchungen sollen in den nächsten Jahren diese Probleme weiter verfolgt werden.

\section{Schriftenverzeichnis}

Armbruster, S., Joachim, H., Lamprecht, K. \& Villinger, E. (1977): Grenzen der Grundwassernutzung im Rhein-Neckar-Raum (Bad.-Württ.). - Z. dt. geol. Ges., 128: 263-96, 15 Abb., 2 Tab.; Hannover.

BARSCH, D. (1976): Das GMK-Schwerpunktprogramm der DFG: Geomorphologische Detailkartierung in der Bundesrepublik. - Z. Geomorph., NF 20: 488-498, 1 Abb., 1 Tab.; Berlin.

Bartz, J. (1959): Zur Gliederung des Pleistozäns im Oberrheingebiet. - Z. dt. geol. Ges., 111: 653-661, 2 Abb., 1 Tab.; Hannover.

Ellenberg, (1978): Vegetation Mitteleuropas mit den Alpen. - 2. Aufl.: 981 S., 499 Abb., 130 Tab.; Stuttgart (Ulmer).

Fezer, F. (1974): Randfluß und Neckarschwemmfächer. - Heidelb. geogr. Arb., 40: H. GraulFestschrift; 167-184, 9 Abb.; Heidelberg.

- (1977): Analysis of River Sediments and Quarternary Ecology. - Catena, 4: 135-138, 2 Abb.; Gießen.

Fietz, A. (1953): Pinus cembra (Zirbelkiefer, Arve) aus dem Diluvium des Oberrheingebietes. Eiszeitalter u. Gegenwart, 3: 47-49; Öhringen.

- , Bortenschlager, S., Felber, H., Heissel, W., Hilscher, H. \& Resch, W. (1970): Der Bänderton von Baumkirchen (Inntal, Tirol). - Z. Gletscherk. Glazialgeol., 6: 5-35; Innsbruck.

FLIRI, F. (1970): Neue entscheidende Radiokarbondaten zur alpinen Würmvereisung aus den Sedimenten der Inntalterrasse (Nordtirol). - Geomorph., 14: 520-21, 9 Abb.; Berlin, Stuttgart.

- (1978): Die Stellung des Bändertonvorkommens von Schabs (Südtirol) in der alpinen Würmchronologie. - Z. Gletscherk. Glazialgeol., 14: 115-118; Innsbruck.

Frenzel, B. (1967): Die Klimaschwankungen des Eiszeitalters. - XII + 291 S.; 107 Abb., 23 Tab.; Braunschweig (Vieweg).

Garnes, K. (1978): Zur Stratigraphie der Weichseleiszeit im zentralen Südnorwegen. In: NagL, H. (Hrsg.): Beiträge zur Quartär- und Landschaftsforschung (Fink-Festschrift), 195-220, 14 Abb.; Wien.

Gerh, M. A. \& Rohde, P. (1972): Weichselian Chronostratigraphy, C14 Dating and Statistics. 24. Int. geol. Congress Canada, Section 12: 27-36, 4 Abb., 3 Tab.; Ottawa.

Grootes, P. M. (1977): Thermal diffusion isotopic enrichment and radiocarbon dating beyond 50000 years B.P. - Diss. Groningen; 221 S., 57 Abb., 45 Tab.; Groningen.

Gross, H. (1964): Das Mittelwürm in Mitteleuropa und den angrenzenden Gebieten. - Eiszeitalter u. Gegenwart, 14: 187-198, 1 Abb.; OOhringen.

Hannss, Ch. (1973): Das Ausmaß der würmzeitlichen Isèretal-Vergletscherung im Lichte neuer Datierungen. - Eiszeitalter u. Gegenwart, 23/24: 100-106, 3 Abb., 2 Tab.; O'hringen.

Hauber, L. \& Barsch, D. (1977): Zur Geologie und pleistozänen Entwicklung des Talkessels von Reigoldswil. - Regio Basiliensis, 18: 85-90, 2 Abb.; Basel. 
KIND, N. V. (1972): Late quaternary climatic changes and glacials events in the old and new world. - Radiocarbon chronology. - Int. geol. Congress Canada, Section 12: 55-61; Ottawa.

Löscher, M. (1979): Erste 14C-Datierungen aus dem Neckarschwemmkegel. - Jber. Mitt. oberrhein. geol. Verein, NF., 60: 175-180, 2 Abb., 1 Tab.; Stuttgart.

Mangold, A. (1892): Die alten Neckarbetten in der Rheinebene. - Abh. hess. geol. LA., 2: 57-114, 2 Taf.; Darmstadt.

PECSI, M. (1978): Paläogeographische Forschung und Vergleich der ungarischen und europäischen Lösse. In: NAGL, H. (Hrsg.): Beiträge zur Quartär- und Landschaftsforschung (Fink-Festschrift), 413-434, $10 \mathrm{Abb}$; Wien.

RoтнsсhiLD, S. (1935): Zur Geschichte der Moore und Wälder im Nordteil der Oberrheinischen Tiefebene. - Beih. bot. Centralblatt, 54, Abt. B.: 140-184; Frankfurt.

SIDKI, K. (1976): Hydrogeologische Untersuchung im Rhein-Neckar-Gebiet (Neckarschwemmfächer). Diss. Fak. Geowiss. - VII+131 S., 29 Beil.; Heidelberg. - [Unveröff.].

Schlüchter, Ch. (1973): Die Münsingenschotter, ein letzteiszeitlicher Schotterkörper im Aaretal südlich Bern. - Bull. Ver. schweiz. Petrol-Geol. u. Ing., 39: 69-78, 54 Abb., 1 Tab., 1 Taf.; Bern.

SchottleR, W. (1906): Erläuterung zur geologischen Karte des Großherzogt. Hessen i. M. $1: 25000$, Blatt Viernheim (Käfertal), 116 S., 1 Abb.; Darmstadt.

Schröder-Lanz, H. (1971): War das Frühwürm (W I) eine selbständige Kaltzeit? - Mitt. Geogr. Ges. München, 56: 173-184, 5 Abb.; München.

Zagwijn, W. \& PAEPE, R. (1968): Die Stratigraphie der weichselzeitlichen Ablagerungen der Niederlande und Belgien. - Eiszeitalter u. Gegenwart, 19: 129-146, 6 Abb.; O'hringen.

Manuskript eingegangen am 1. 3. 1980. 
Tafel 1
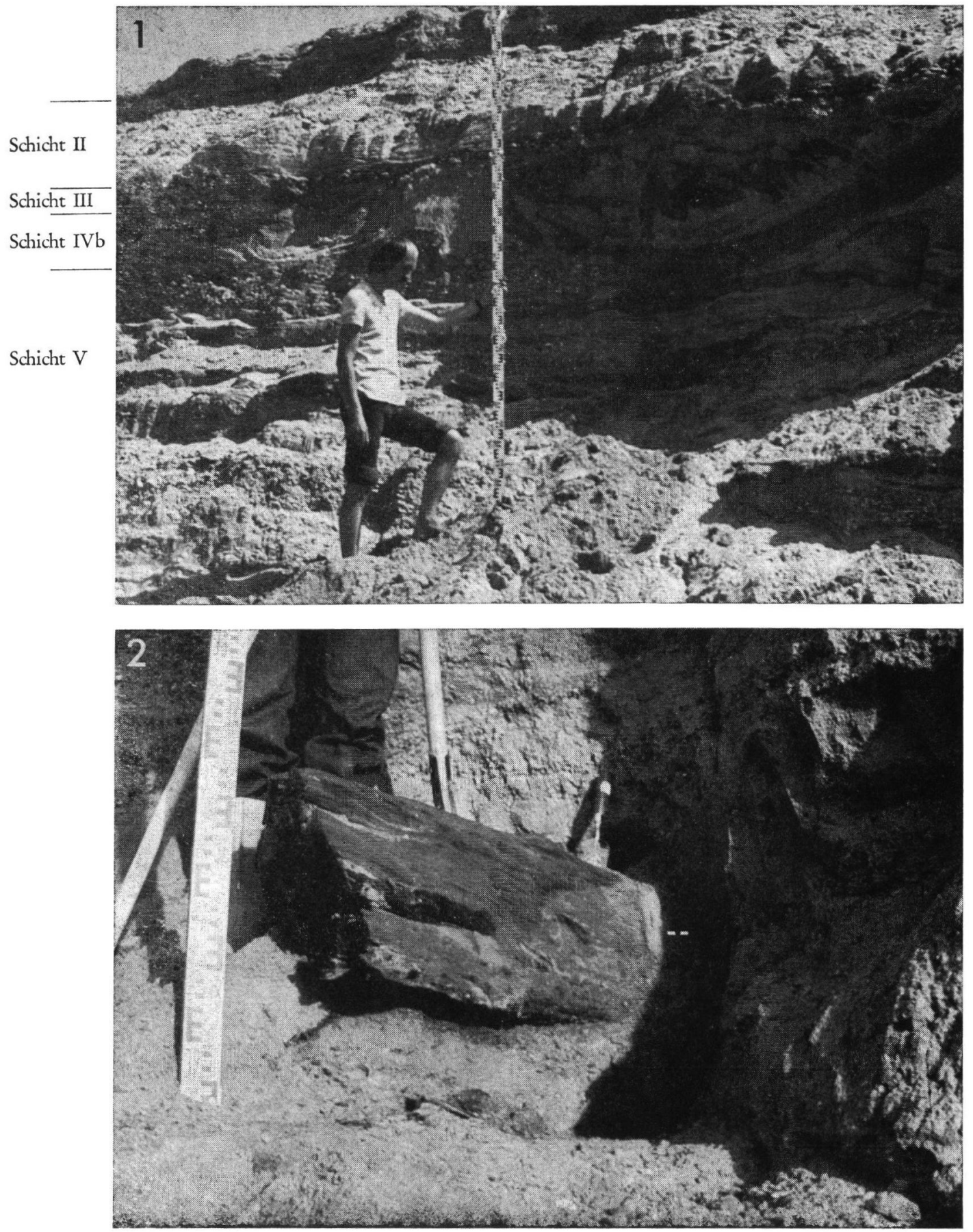

Fig. 1: Kryoturbationserscheinungen in Schicht IV b in der Kiesgrube Heckmann nordwestlich Mannheim-Wallstadt. Photo: M. Löscher.

Fig. 2: Holzfunde an der Obergrenze von Schicht V in der Kiesgrube Heckmann nordwestlich Mannheim-Wallstadt. Photo: M. Löscher. 